\title{
Norms Enforcement as a Coordination Strategy in Ubiquitous Environments
}

\author{
Ismail Khalil Ibrahim, Reinhard Kronsteiner, and Gabriele Kotsis \\ Institute of Telecooperation, Johannes Kepler University Linz, \\ Altenberger Str. 69, A-4040 Linz, Austria \\ Tel.: +43-73224689888, Fax: +43-73224689829 \\ \{ismail, reinhard, gk\}@tk.uni-linz.ac.at
}

\begin{abstract}
Ubiquitous environments are characterized by their openness, dynamicity and autonomy. Electronic institutions are the agents' counterpart of human organizations, which are specifically designed for providing support, trust, and legitimacy in ubiquitous applications. In this paper, we propose an algorithm for norms enforcement in electronic institutions as a coordination strategy in ubiquitous environments by introducing substitution rules that map the norms set for the electronic institution to normative rules that can be implemented in the protocols to govern agents' interactions in ubiquitous environments.
\end{abstract}

\section{Introduction}

In ubiquitous environments [11], a set of mobile (mostly autonomous) entities, each with limited resources and knowledge, needs to interact with each other and users to achieve a common goal within a specific context. This interaction is generally characterized by 1) openness, describing the possibilities that these mobile entities can join or leave the environment, 2) flexibility indicating the degree the mobile entities are restricted by the environment rules and norms, 3) stability defining the predictability of the consequences of actions and 4) trustfulness specifying the extent to which users may trust the environment

The infrastructure that supports this ubiquitous environment must meet two requirements, first the internal autonomy requirement of participating entities in which interaction and structure of the environment must be represented independently from the internal design of the participating entities and second the collaboration autonomy requirement in which activities and interaction in the environment must be specified without completely fixing in advance the interaction structure.

In order to achieve these requirements, we argue that open ubiquitous environments can be modeled as electronic institutions where a vast amount of heterogeneous software and human agents interact in order to satisfy common and/or individual goals.

Electronic Institutions are the agents' counterpart of human organizations, which are specifically designed for providing support, trust, and legitimacy in agent mediated interactions [2][3][5][7]. They can be considered as computational frameworks that function the same way our human organizations function providing services and rules for software agents to meet in a way to create trust, prevent fraud 
and reduce deception by verifying regulations which result in maintaining norms and insuring that all parties are conforming to these norms

Agents in this sense are computational entities that are situated in some environment and capable of flexible, autonomous action in that environment in order to meet its design objectives [12]. They can accept a task, negotiate or reject it. They should be capable to communicate, to coordinate, and to cooperate with other agents in order to solve a problem.

Norms in the context of electronic institutions are expectations about what behaviour, thoughts, or feelings are appropriate within a given group (of agents) within a given context (environment).

The study and modelling of norms in electronic institutions has attracted the interest of researchers from different disciplines such as sociology, economics, philosophy, and computer science.

Two approaches have been advocated for the design and modelling of norms in electronic institutions: in the cooperative normative behaviour [1][3][5], norms are defined implicitly through the behaviour of the agents and depends on how agents function in the institution; in the coordination strategy [10], norms are defined explicitly and the agents in the institution have to comply to these norms.

Most of the cooperative normative behaviour model is based on the assumption that different agents in the institution have some common goal or intention and this common goal enforces some type of cooperative behaviour on all agents in the institution. This means that the rules and regulations to which agents adapt their behaviour are hard wired in the protocols used by the agents to interact with each other. In the coordination model, actions of agents in the electronic institution are either rational or norm guided. In this case, norms are nothing but instruments of individual, collective, or generic optimization and to accept norms, as aïmotivational mechanism is not to violate methodological individualism, or to deny the importance of rational choice. In this case, the outcome of agent actions is a compromise between what the norm prescribes and what rationality dictates.

In this paper, we address the problem of norm enforcement in ubiquitous environments. We propose an algorithm for norm compliance checking by introducing substitution rules that map the norms set for the electronic institution as values to normative rules that can be implemented in the protocols to govern agents' interactions.

\section{Norms as a Coordination Strategy}

One of the most important challenges in open environments like ubiquitous computing, is the specification of mechanisms through which perspective participants can evaluate the characteristics and objectives of the environment rules in order to decide about participation.

Currently, in ubiquitous applications, agents are simply designed from scratch so that their behaviour complies with the behaviour described by the role(s) it will take up in the environment. Comprehensive solutions for this point require complex agents that are able to reason about their own objectives and desires and thus decide and negotiate their participation. As a first step for this is the comparison between the specification of agents and roles to determine whether an agent is suitable to enforce norms [2].

An important aspect concerning norm enforcement is that of modifying the agent internal architecture or beliefs to include the characteristics of the assumed role(s). A 
possible solution for this has been proposed by [4], which extends agents with an interface to the society. This interface prevents any action not allowed by the role definition. However, it does not ensure the proactive behaviour expected from the role and is not flexible enough to incorporate different enacting styles, capabilities and requirements of the agents. It actually makes the actual agent "invisible" to the society and only its enactment of the role behaviour apparent in the society. We think that the consequence of an agent adopting a role is more drastic than this. The actual agent behaviour must often be modified according to the goals, norms and rights specified by the role.

In the following, we assume that agents have goals of their own, and are able to form (either by design or deliberation) plans to achieve those goals. These assumptions are consistent with the agent view and can be seen as a minimal agent definition. Here we describe agent societies from a global perspective, rather than from the perspective of the individual agents. Even though agents will take many roles simultaneously and along their life cycles, from the perspective of the society, each agent is a different individual. From our perspective, it is up to the agent how to manage and prioritize its goals. That is by assuming a role; the agent will receive the objectives from that role. How the agent will handle those objectives, whether it interprets them as goals or as norms, what priority it gives them, is up to the agent itself. However, the society model is based on the assumption that agents that take up roles are expected to eventually realize the assumed objectives.

Nevertheless, societies are concerned with judging the attitudes of the different areas and how those will affect the performance of the role. That is, the society will not look at the agent as a whole but at how a certain area is acting.

Agent literature discusses extensively different types of social attitudes of agents: selfish, altruistic, honest, and dishonest.

Different types of agents result in different role performances, because the way an agent will plan its goals, which is dependent on his social attitude, influences the realization of its role objectives and the fulfillment of the role norms. For instance, some types of agents will only attempt to achieve the goals of their adopted roles and forget their own private goals, while others will only attempt to achieve the goals from the role after all their own goals have been satisfied. Furthermore, the relations between agent plans and role objectives, and of agent goals and sub objectives must be considered

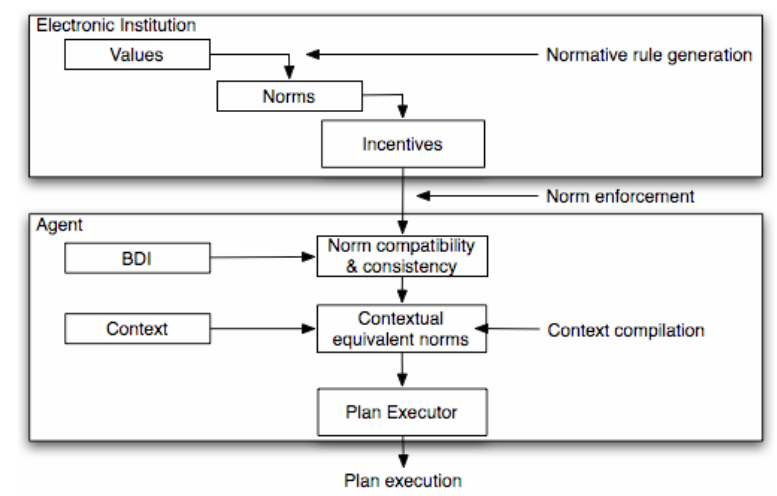

Fig. 1. Architecture of norm enforcement 
In our approach [8], we suppose that we have a set of value definitions stored in the normative structure of a certain electronic institution. We consider the problem of given a norm $N$ that is captured from the normative structure of the institution through semantics, how to find a rewriting $N^{\prime}$ for it using one or more of the available normative rules such that $N$ is contained in $N^{\prime}$ (i.e., the value of $N$ is a subset of $N^{\prime}$ ). It is well known that containment implies equivalence.

We consider the problem of verifying partial compliance and verifying complete compliance of agents to norms set for the electronic institution.

A partial compliance of a norm $N$ using a set of normative rules $R$ is a conjunctive norm $N^{\prime}$ whose head is a norm literal, such that $N^{\prime} \subseteq N$ and the body of $N^{\prime}$ contains one or more normative rule literals defined in $R$ (it may contain value literals as well).

A complete compliance of $N$ using $R$ is a partial compliance of $N$ using $R$ whose body contains normative rules and built in predicates only (it may not contain value literals).

In our approach to norm compliance, we refer to the architecture depicted in figure 1, in which four phases are identified:

1. The normative rules generation phase where values $V$ are expressed in terms of the normative rules $R$. The result of this phase is a set of normative rules $R$ that contain all the necessary information for norm enforcement phase.

2. In the context compilation phase, the context [7] is used to compute a set of contextually equivalent norms $(C E N)$. These contextually equivalent norms $C E N$ are filtered and stored for later use.

3. The norm compliance phase, which uses the contextually equivalent norms to decide whether, norm $N$ is consistent to the set of norms defined for the electronic institution. This phase uses the set of normative rules generated earlier to rewrite the norm in such a way to generate an equivalent norm $N^{\prime}$ that produce the same effect as the original one but defined over the component normative rules.

4. The plan execution phase, where a norm $N$ defined over the values is verified to comply to the electronic institution regulations and rules

\subsection{Example Scenario (Adapted from [9])}

In order to understand the system architecture, we will model the mobile auction scenario (mobile flea market) we presented in [9] as an institution. This example illustrates a number of important characteristics that is quite common to ubiquitious environments and must be taken into account in the development of an agent based computational market [9]. There are multiple services available from a number of agents representing independent participants. Multiple agents offer broadly similar services. The services themselves are described by multiple attributes (e.g. price, quality and delivery time). The services available change over time (new services may become available, or agents may alter the way in which existing services are offered). Services may differ in terms of the number and heterogeneity of the tasks involved in the delivery of the service and in their degree of independence and the type and frequency of interactions between different customers changes while the service is being delivered.

For the sake of clarification, let us assume that the mobile flea market sets the following vision as a value, 


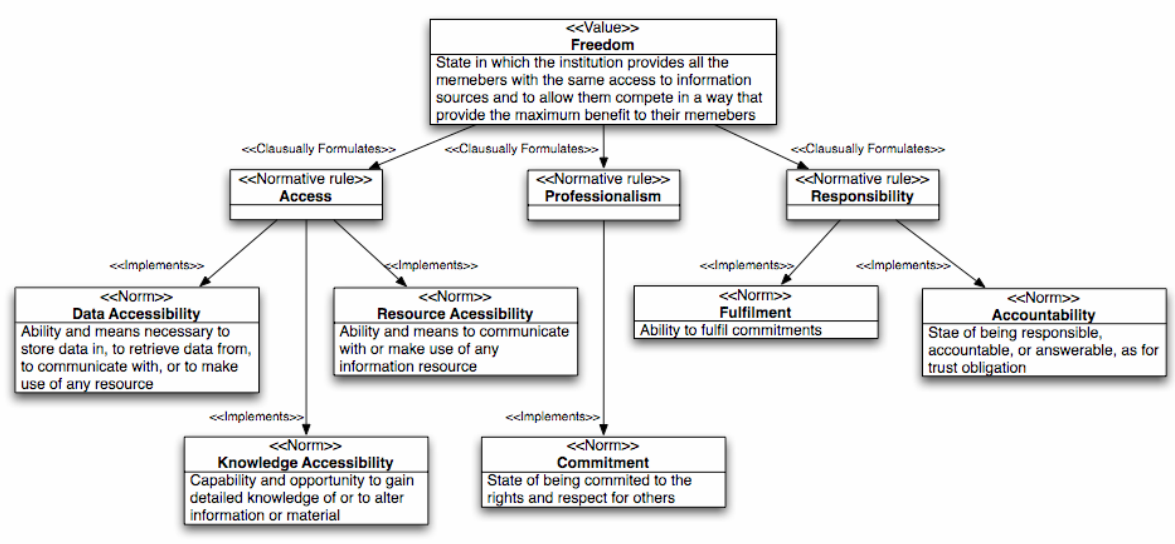

Fig. 2. Normative structure of the example scenario

"Fostering the highest standard of free competition amongst those for whom the institution is responsible"

Stakeholders in the institution are sellers, buyers, auctioneers, market owners, and accountants. The objective of the market owners is to provide a fair set up of the market where sellers sell their goods at the highest price, buyers find what they are looking for in an efficient way, and buy products with the cheapest price. All expect to have a win -win situation.

As we see, the notion of free competition is a very general concept and can be interpreted according to different stakeholder interests and intentions. This means that the value of fair competition can be translated into three normative rules; one is related to the access to resources within the institution; and the second is concerned with the professionalism with which the institution is operating; and the third is responsible management. These three normative rules can be further translated into low-level norms. The normative structure of the example is depicted in Figure 2.

Let us define two values for our conference institution:

Accountability(Sellers, Buyers, Products)

Freedom(Access, Professionalism, Responsibility)

Suppose the electronic institution maintains two normative rules:

Access(Data, Resources, Knowledge) $\leftarrow$ Accountability (Seller, -, -, -)

Responsibility(Fulfillment, Commitment) $\leftarrow$ Accountability (-,-,Buyers)

And the following set of norms:

- $\mathrm{N}_{1}$ : A trading agent winning a bidding round within an auction is obliged to subsequently pay for the acquired good.

- $\mathrm{N}_{2}$ : The seller agent is committed not to sell goods or products, which had been already sold.

- $\mathrm{N}_{3}$ : The seller agent is responsible of delivering goods or products to the buying agents.

- $\mathrm{N}_{4}$ : No bids after the deadline. 


\subsection{Normative Rules Generation}

In this phase, the values are expressed in terms of the normative rules. The result is a set of rules that contains all the information needed for the norm enforcement phase. The generator can be thought as a specific model checker for normative rules.

Consider a normative rule of the form:

$\mathrm{R} \leftarrow \mathrm{V} 1, \ldots, \mathrm{Vn}, \mathrm{C} 1, \ldots . ., \mathrm{Cm}$,

where

$V_{1}, \ldots \ldots, V_{\mathrm{n}}$ are value literals and

$C_{1}, \ldots \ldots, C_{\mathrm{m}}$ are constraints.

Let $N_{1}, \ldots \ldots \ldots, N_{1}$ be the distinguished variables and

$R_{1}, \ldots \ldots ., R_{\mathrm{m}}$ be the rest of variables.

To avoid having to deal with the existentially quantified variables $X_{1}, \ldots \ldots \ldots, X_{1}$, we skolemize the normative rule by substituting for all $Y_{\mathrm{j}}$ a different skolem term of the form $f_{\mathrm{j}}\left(N_{1}, \ldots \ldots \ldots, N_{\mathrm{l}}\right)$. Let $\theta$ be the substitution that skomelizes all such $R \mathrm{~s}$.

Since all the normative rules predicates in $\mathrm{R}$ are completely defined by its body, then the normative rule implies the formula:

$\left(\forall N_{1}, \ldots, N_{\mathrm{l}}\right)\left(R \rightarrow\left(\exists R_{1}, \ldots, R_{\mathrm{m}}\right)\left(V_{1} \wedge \ldots \wedge V_{\mathrm{n}}\right)\right.$.

After skolemization, we derive $\mathrm{n}$ substitution rules below,

$\mathrm{V} 1 \theta \leftarrow \mathrm{R}$

$\mathrm{Vn} \theta \leftarrow \mathrm{R}$

where

$\theta=\{\mathrm{Ni} \leftarrow \mathrm{fi}(\mathrm{Ni}, \ldots \ldots . \mathrm{Nl})\} 1 \leq \mathrm{i} \leq \mathrm{m}$ is a substitution rule and $\mathrm{f} 1, \ldots \ldots, \mathrm{fm}$ are $1-$ ary skolem functions with arguments $\mathrm{N} 1, \ldots . . . \mathrm{Nl}$.

For each substitution rule the head is a value literal, and every variable in the head also appears in the body.

The skolem function $f_{\mathrm{i}}\left(N_{1}, \ldots \ldots N_{1}\right)$ is a mapping or transformation from the normative rule to the value that assigns to each variable in $R\left(\right.$ i.e., $\left.N_{1}, \ldots \ldots N_{\mathrm{l}}\right)$ a variable of $\mathrm{V}$ such that $f_{\mathrm{i}}\left(N_{\mathrm{i}}, \ldots \ldots . N_{\mathrm{l}}\right) \in V$. The normative rule is the domain of the skolem function and the value is the co-domain.

\subsection{Norm Enforcement}

Given a norm $N$ defined over the set of values, a set $R$ of normative rules $\left\{R_{1}, \ldots \ldots, R_{\mathrm{k}}\right\}$ and a set $\theta$ of substitution rules $\left\{\theta_{1} \ldots \ldots \theta_{\mathrm{n}}\right\}$.

Our goal is to find an equivalent norm $N^{\top}$ that is compliant to the set of norms defined for the electronic institution. A norm $N^{\prime}$ of $N$ using $R$ can be obtained by rewriting $N$ using $R$ in such a way that the resulting norm contain one or more normative rules predicates defined in $R$ and produce the same effect as the original norm for any given electronic institution.

Norm Enforcement process can be done in two steps:

The first step is to determine the valid norm substitutions. Norm substitution is the process of assigning to each value literal in the body of the norm the substitution rule(s) $\left\{\theta_{1} \ldots \ldots \theta_{\mathrm{k}}\right\}$ that is defined in $S$ for that literal. A substitution applied to the rule is the replacement of each variable in the rule by either a variable or a constant, while 
a substitution applied to a norm is the replacement of each value literal by the substitution rules derived from the normative rules definitions for those literals.

The substitution is evaluated then for each variable in the head of the norm with respect to each substitution rule.

Proposition (1) determines when the substitution is valid and proposes the way to rewrite the norm.

\section{Proposition (1)}

A substitution is valid if all the distinguished variables that appear in the head of the norm are substituted by the same variable in the substitution rule not by a skolem term

A valid substitution means that the distinguished variable of the norm maps into the head of the normative rule that is defined by the substituted value and hence means that this distinguished variable comply to the normative rule as indicated by in proposition (2)

\section{Proposition (2)}

For each $n_{i} \subseteq V_{i}$ such that $V i \theta j \leftarrow R j$

If $\sum_{\theta_{j}}^{V_{i}} n_{i}$ is a valid substitution for Vi in $\theta j$ Then $n_{i}$ comply to $R_{j}$

Returning to proposition 1 . The valid substitution for $x_{\mathrm{i}}$ means that $n_{\mathrm{i}}$ of the norm $N$ maps into the head of the normative rule $R_{\mathrm{j}}$ defined by $V_{\mathrm{i}}$ and hence $N$ complies partially to $R_{\mathrm{j}}$

Conversely, if $\sum_{\theta_{j}}^{V_{i}} n_{i}$ is not a valid substitution, then $n_{\mathrm{i}}$ doesn't map into the head of the normative rule $R_{\mathrm{j}}$ defined by $V_{\mathrm{i}}$ and hence there is no partial compliance for $N$ in $R_{\mathrm{j}}$

The second step in the norm rewriting process is to construct the set of all the possible rewritings of the norm. This can be simplified by considering the set of all the substitution rules for each value in the body of the norm as an $(m \times n)$ matrix where $m$ is the number of the distinguished variables in the head of the norm and $n$ is the number of the substitution rules defined in $S$ for the value indicated by the matrix.

A valid substitution is considered to be 1 or TRUE and invalid substitution as 0 or FALSE to simplify the notation.

For example, if we have one value in the body of the norm, two substitution rules defined in $S$ for that value, and three distinguished variables in the head.

So we construct the $(3 \times 2)$ matrix for the norm as follows

$$
\begin{array}{cccc}
\sum_{\theta_{1}}^{V_{1}} n_{1} & \sum^{V_{1}} n_{2} & \sum^{V_{1}} n_{3} \\
\sum_{\theta_{2}} & 1 & 1 & 0 \\
& 1 & 0 & 1
\end{array}
$$


Now to compute the possible rewritings of the norm described by this matrix, we need to evaluate the valid substitutions w.r.t each distinguished variable in the head of the norm.

\section{Proposition (3)}

A value literal $V_{i}$ in the body of a norm $N$ can be replaced by the normative rule literal $R_{j}$ defined in $R$ if

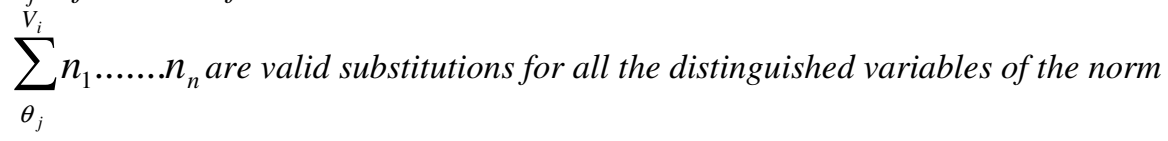

This means that if there is an all 1s row in our matrix, then all the distinguished variables are compliant to the normative rules and the value literal corresponding to that matrix can be replaced by the normative rule literal indicated by the row.

As suggested by proposition (3), any one of the normative rules can't replace the value literal in the norm since there is no all $1 \mathrm{~s}$ row in the its matrix.

Recalling proposition (2) where a valid substitution for any distinguished variable means that the normative rule defined in the substitution rule is partially compliant to the norm.

So we need to evaluate the partial compliance for the distinguished variables in the valid substitution matrix.

\section{Proposition (4)}

The conjunction of all the partial compliances for a norm is a rewriting if there exists

$$
\sum_{\theta_{j}}^{V_{i}} n_{i} \text { that is a valid substitution for all the distinguished variables in the }
$$

substitution matrix.

For the norm in example 4 we have the following partial compliances:

$$
\begin{aligned}
& \text { For } n_{1}: R_{1} \vee R_{2} \\
& \text { For } n_{2}: R_{1} \\
& \text { For } n_{3}: R_{2}
\end{aligned}
$$

Since there is partial compliance for all the distinguished variables in the norm. Then the conjunction of these partial compliances is a rewriting and we get the following rewriting

$$
N\left(n_{1}, n_{2}, n_{3}\right) \leftarrow R_{1}\left(n_{1}, n_{2}, R_{1}, R_{2}\right), R_{2}\left(n_{1}, R_{3}, n_{3}, R_{4}\right)
$$

For this norm we have the following two valid substitutions matrices:

$$
\begin{array}{cccccc}
\sum_{\theta_{1}}^{V_{1}} n_{1} & \sum^{V_{1}} n_{2} & & \sum^{V_{2}} n_{1} & \sum^{V_{2}} n_{2} \\
\sum_{\theta_{2}} & 1 & 1 & \sum_{\theta_{1}} & 1 & 1 \\
& 1 & 0 & \sum_{\theta_{2}} & 0 & 0
\end{array}
$$

From the substitution matrices above we notice that $R_{1}$ can replace each $V_{1}, V_{2}$ in the norm so the norm can be rewritten as follows: 
We also get the same rewriting from the partial compliances for this norm. For this norm we have the following two valid substitutions matrices:

$$
\begin{aligned}
& \sum^{V_{1}} n_{1} \quad \sum^{V_{1}} n_{2} \\
& \sum^{V_{2}} n_{1} \quad \sum^{V_{2}} n_{2}
\end{aligned}
$$

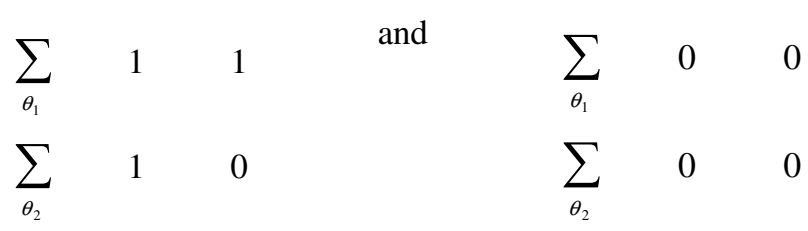

Again we notice that $R_{1}$ can replace $V_{1}$ in the norm, while there is no normative rule that can replace $V_{2}$, hence the following is a rewriting of the norm.

$N\left(n_{1}, n_{2}\right) \leftarrow R_{1}\left(n_{1}, n_{2}, R_{2}, R_{3}\right), V_{1}\left(n_{1}, " \ldots . ", n_{3}, R_{4}\right)$.

There is no rewriting that can be derived from the partial compliance since $n_{1}$ and $n_{2}$ have no valid substitutions in $R_{2}$.

\section{Conclusion and Future Work}

Agents are either always comply with norms or autonomously choose whether to do so or not based on the utility or situation.

This may cause conflicts between the electronic institution goals and the goals of agents within it. On the one hand, if agents are assumed to comply to norms, this may decrease the opportunities agents have to achieve their goals. On the other hands, if agents choose whether to comply to norms set by the institution or not, although agent goals may be satisfied, the electronic institution becomes unpredictable when all agents choose not to comply to norms and consequently result in violations of the overall essence of the organization

The purpose of the project from which this research paper stems is to build a complete monitoring system for electronic markets. Our objective is to integrate seamlessly the cooperation and coordination models as a basis for modelling electronic institutions.

\section{References}

[1] C. Castelfranchi, F. Dignum, C. Jonker, and J. Treur, "Deliberative Normative Agents: Principles and Architectures", Proc. of the 6th Workshop on Agent Theories, Architectures and Languages, ATAL'99, Orlando, 1999, pp. 206-220.

[2] M. Dastani, V. Dignum and F. Dignum, "Organizations and Normative Agents", Proceedings of first Eurasian Conference on Advances in Information and Communication Technology, Teheran Iran, 2002, pp. 982-989

[3] F. Dignum, "Abstract Norms and Electronic Institutions", Proceedings of the Int. Workshop on Regulated Agent-based social Systems: Theories and Applications RASTA'02, Bologna Italy, 2002

[4] V. Dignum, A Model for Organizational Interaction based on Agents, founded in Logic, $\mathrm{PhD}$ thesis at Utrecht University 2004. SIKS Dissertation Series No 2004-1 
[5] M. Esteva, J. Padget and C. Sierra, "Formalizing a Language for Institutions and Norms", J.J.Ch. Meyer, and M. Tambe, (eds.) Intelligent Agents VIII, Vol. 2333 LNAI, Springer 2001

[6] I.K. Ibrahim, E. Weippl, F. Dignum, and W. Schwinger, "Modelling Norms in Electronic Institutions" Proc. of the Workshop on Agent-Based Simulation, Passau, Germany 2002.

[7] I.K. Ibrahim, G. Kotsis and W. Schwinger, "Mapping Abstraction of Norms in Electronic Institutions", Proceedings of the $12^{\text {th }}$ IEEE International Workshop on Enabling Technologies: Infrastructure for Collaborative Enterprises WETICE'03, Linz, 2003, pp. 30-35

[8] I.K. Ibrahim, G. Kotsis and R. Kronsteiner, „Substitution rules of norm-compliance in electronic institutions", Proc. Of International workshop on enabling technologies: Infrastrucutre for collaborative enterpricses, Modena June 2004.

[9] I.K. Ibrahim, R. Kronsteiner and G. Kotsis, "Agent-based mobile auctions: The flea market scenario", Proc. Of 2004 Research conference in innovations in information technology (IIT2004), Dubai Oct. 2004.

[10] F. López y López, M. Luck, M. and M. d'Inverno, “Constraining autonomy through norms" Proc. of the 1st Joint Conference on Autonomous Agents \& Multiagent Systems, AAMAS 2002 ACM, 2002, pp. 674-681

[11] J. Ma, L.T. Yang, B.O. Apduhan, R. Huang, L. Barolli and M. Takizawa, "Towards a smart world and ubiquitous intelligence: a walktrough from smart things to smart hyperspaces und UbicKids", Journal of pervasive computing and communications 1(1), Troubador publishing, March 2005.

[12] J. Vázquez-Salceda and F. Dignum,. „Modelling Electronic Organizations“, V. Marik, J. Mueller and M. Pechoucek, (eds.), Proceedings of Central and East European Conference in Multi-Agent Systems CEEMAS’03, Springer, Prague, 2003 